\section{Estudo descritivo das alterações posturais de coluna vertebral em escolares de 10 a 16 anos de idade. Tangará-SC, 2004}

\section{Descriptive study of backbone postural changes in 10 to 16 year-old schoolchildren. Tangará-SC, Brazil, 2004}

\section{Raquel Cristina Martelli \\ Jefferson Traebert ${ }^{2}$}

'Aluna de Mestrado em Saúde Coletiva, Universidade do Oeste de Santa Catarina/ UNOESC

${ }^{2}$ Professor Doutor, Mestrado em Saúde Coletiva, Universidade do Oeste de Santa Catarina/ UNOESC

\section{Resumo}

Objetivo: Conhecer a prevalência de alterações posturais em coluna vertebral e fatores associados em escolares de 10 a 16 anos do município de Tangará, SC. Métodos: Estudo descritivo transversal. Os dados foram coletados através de avaliação fisioterapêutica individual e de entrevista estruturada. Para a obtenção das alterações posturais, os escolares foram avaliados em três posições anatômicas, baseando-se na posição de ombros/escápulas, coluna, membros superiores, nível pélvico e abdômen. Coletou-se também o peso e a estatura dos escolares. Na entrevista foram questionadas a idade, escolaridade e a dominância do indivíduo, a renda familiar e a escolaridade dos pais. Para o estudo de associação entre as variáveis utilizou-se o teste do Qui-Quadrado ou Exato de Fisher. Resultados: A prevalência de alterações posturais foi de $28,2 \%$. As duas alterações mais prevalentes foram a hiperlordose com $20,3 \%$ e a hipercifose com $11,0 \%$. Uma menor estatura mostrou-se estatisticamente associada à prevalência de alterações posturais. Além disso, uma menor estatura e um menor peso corporal mostraram-se estatisticamente associados à ocorrência de hiperlordose. Conclusões: A prevalência de alterações posturais de coluna entre os escolares de Tangará, SC, foi de $28,2 \%$. Os indivíduos mais baixos e com menor peso corporal apresentaram mais alterações posturais em relação aos seus colegas mais altos e com maior peso corporal.

Palavras-chave: Alteração postural. Coluna vertebral. Prevalência. Escolares. 
Abstract

Objective: To find out the prevalence of and factors associated with backbone postural changes in 10 to 16 year-old schoolchildren of Tangará, SC, Brazil. Methods: A cross-sectional study was carried out. Clinical physiotherapeutic examinations and interviews were performed. Students' weight and height were also collected. Students' age, education and dominance, as well as schooling of parents and family income were asked during the interview. Chisquare and Fisher's exact test were used to observe the associations between study variables. Results: The prevalence of backbone postural changes was $28.2 \%$. The two most frequent changes were high concavity with $20.3 \%$ and high kyphosis with $11.0 \%$. Shorter height students showed a higher prevalence of backbone postural changes. Moreover, shorter height and lower body weight were statistically associated with a higher occurrence of high concavity. Conclusions: The prevalence of backbone postural changes was $28.2 \%$. Individuals with lower height and body weight showed a higher prevalence of backbone postural alterations.

Keywords: Postural alteration. Backbone. Prevalence. Schoolchildren.

\section{Introdução}

Problemas físicos que podem acometer crianças e adolescentes e que têm início na fase de crescimento constituem fator de risco para disfunções de coluna vertebral irreversíveis na fase adulta ${ }^{1-3}$.

As alterações posturais relacionadas às posturas inadequadas são distúrbios anátomo-fisiológicos que se manifestam geralmente na fase de adolescência e préadolescência, pois é o período em que há o estirão de crescimento ${ }^{2}$.

Dados epidemiológicos apontam para uma alta prevalência de alterações posturais de coluna entre crianças e adolescentes. Estudo envolvendo escolares de 6 a 17 anos de idade mostrou que a hipercifose estava presente em $20,9 \%$, com predominância no sexo masculino ${ }^{4}$. Outro estudo também com escolares mostrou que $84,9 \%$ das crianças de 7 a 14 anos apresentavam protrusão de ombros e hipercifose dorsal ${ }^{5}$.

É importante a detecção precoce e a prevenção desses problemas, associados às orientações quanto à postura correta, pois a maioria dos problemas é decorrente de etiologia idiopática e devida à má postura durante as atividades de vida diária $^{3}$.

Geralmente considera-se a postura anormal como o fator etiológico principal de condições dolorosas e incapacitantes ${ }^{6}$. Todavia, os indivíduos principalmente na fase de crescimento, podem apresentar uma postura anormal, mas devido à boa flexibilidade não apresentam condições dolorosas. Por outro lado, há relatos de que pode haver indivíduos com boa postura aparente, mas com limitação anátomofuncional e presença de condições dolorosas? ${ }^{7}$.

A postura adequada na infância ou a correção precoce de desvios posturais nessa fase possibilitam padrões posturais corretos na vida adulta, pois esse período é da maior importância para o desenvolvimento músculo-esquelético do indivíduo, com maior probabilidade de prevenção e tratamento dessas alterações posturais na 
coluna vertebral. Por outro lado, na maturidade podem se tornar problemas irreversíveis e sem tratamento específico ${ }^{1}$. A idade escolar compreende a fase ideal para recuperar disfunções da coluna de maneira eficaz; após esse período, o prognóstico torna-se mais difícil e o tratamento mais prolongado $^{3}$.

O objetivo desse estudo foi conhecer a prevalência de alterações posturais na coluna vertebral e fatores associados em escolares de 10 a 16 anos do município de Tangará, SC, em 2004.

\section{Metodologia}

Foi realizado um estudo transversal incluindo todos os escolares de 10 a 16 anos de idade do município de Tangará, SC, em 2004. Trata-se de um município de cerca de 8 mil habitantes, localizado no vale do Rio do Peixe, a $420 \mathrm{~km}$ de distância de Florianópolis, capital do Estado de Santa Catarina.

$\mathrm{Na}$ faixa etária de interesse deste estudo havia 420 alunos matriculados nas duas escolas do município. Os secretários municipais de Educação e Saúde autorizaram a realização do estudo e forneceram dados referentes ao número de escolares por idade matriculados e endereços das escolas.

$\mathrm{O}$ projeto de pesquisa foi submetido e aprovado pelo Comitê de Ética em Pesquisa da Universidade de Passo Fundo, RS. Uma carta de consentimento livre e esclarecido foi enviada aos pais, conforme resolução específica do Conselho Nacional de Saúde, explicando os objetivos, as características e a importância do estudo e solicitando a autorização dos pais para participação de seu (sua) filho (a) no estudo. Após a coleta de dados, todos os pais foram informados sobre as alterações posturais em seus filhos, detectadas no estudo. Além disso, receberam orientação por escrito para procurarem o serviço de saúde do município para uma avaliação clínica mais detalhada de seu(sua) filho(a).

Os exames clínicos foram realizados por uma fisioterapeuta auxiliada por um monitor e um anotador. Previamente ao trabalho de campo, a equipe participou de um exercício de calibração envolvendo 20 escolares de faixa-etária diferente do estudo, conforme metodologia descrita na literatura ${ }^{8}$.

A obtenção de dados clínicos baseouse na avaliação postural individual com o objetivo de verificar a prevalência das alterações posturais nos escolares, através dos critérios propostos por Kendall, McCreary Tais critérios possibilitam determinar as alterações posturais do tipo hipercifose, hiperlordose, escoliose com evidências estruturais, escoliose funcional, cifoescoliose estrutural, cifoescoliose funcional, lordoescoliose funcional, lordoescoliose estrutural e postura cifose-lordose. Obteve-se ainda a altura em metros e o peso corporal em quilogramas.

As duas escolas participantes do estudo foram visitadas em dois momentos distintos. Foram excluídos do estudo os escolares que apresentaram alguma deficiência física que impossibilitasse o exame, que não desejaram participar e não trouxeram o consentimento livre e esclarecido assinado pelos pais ou responsáveis. O exame e a entrevista foram realizados consecutivamente, em uma sala de aula com ampla iluminação natural, além da iluminação artificial com lâmpadas fluorescentes, em fluxo contínuo organizado pela monitora. O escolar se posicionava em frente ao examinador, voltado para uma janela. Foi adotada a postura de pé, utilizando-se como ponto de referência um fio de prumo. Na parede posterior ao fio de prumo, foi utilizado um painel quadriculado denominado posturógrafo para possibilitar melhor visualização de desníveis de ombro e cintura pélvica na postura anterior. Além dos anteriormente citados, os seguintes materiais foram utilizados para avaliação dos escolares: pranchas de postura (tábuas de madeira compensada com impressões podálicas); fio de prumo que foi suspenso de uma barra acima da cabeça, e o peso do prumo suspenso em linha com o ponto de base-padrão; lápis 
dermatográfico para marcar os processos espinhosos a fim de observar a posição da coluna em casos de desvio lateral. Os escolares usaram roupas adequadas para o exame, como short e blusa para as meninas e short para os meninos. O examinador ditava os códigos que eram anotados pelo anotador. Durante o levantamento epidemiológico foram reexaminados $10 \%$ da população de estudo, com o objetivo de se aferir o grau de reprodutibilidade dos critérios de diagnóstico.

Os dados obtidos através da entrevista estruturada com os escolares, incluíram questões sobre idade (em anos completos), escolaridade (a série escolar de freqüência) e a dominância (qual a mão que mais utilizava para escrever) do escolar, além da renda familiar (em reais) e escolaridade do pai e da mãe (até que séries estudaram).

Para os testes de associação entre as variáveis, foi considerada em um primeiro momento, a presença de quaisquer alterações posturais em coluna vertebral. Em um segundo momento, as alterações posturais mais prevalentes foram consideradas para os estudos de associação. As variáveis explanatórias categóricas foram o sexo (masculino; feminino) e a dominância (destro; sinistro). A idade e escolaridade dos pais, renda familiar e peso corporal em $\mathrm{kg}$ foram dicotomizados na mediana da distribuição de freqüência. A distribuição dos valores da altura do indiví- duo em metros foi dicotomizada na média. Foi calculado o Índice de Massa Corpórea - IMC (peso/altura²) que foi dicotomizado na mediana da distribuição. Utilizou-se o teste do Qui- quadrado ou Exato de Fisher para testar a associação entre as variáveis de estudo com nível de significância $\mathrm{p}<0,05$.

\section{Resultados}

Foram examinados e entrevistados 344 escolares, proporcionando uma taxa de resposta de $81,9 \%$. Os principais motivos que contribuíram para a não participação foram faltas às aulas nos dois dias de exame e negação do próprio escolar em ser avaliado. O nível de reprodutibilidade da examinadora manteve-se adequado, com um valor mínimo de kappa de 0,70.

A prevalência de alterações posturais encontrada foi $28,2 \%$ (IC $95 \% 23,4-32,9$ ). As duas alterações mais prevalentes foram a hiperlordose com 20,3\% (IC 95\% 16,1-24,5) e hipercifose com 11,0\% (IC 95\% 7,7-14,3). Outros tipos de alterações posturais foram menos freqüentes (Tabela 1).

A Tabela 2 mostra os resultados do teste de associação entre a presença de qualquer tipo de alteração postural e variáveis sociodemográficas e antropométricas. Apenas a estatura do escolar mostrou-se estatisticamente associada com a prevalência de alterações posturais. Escolares

Tabela 1 - Prevalência de alterações posturais de coluna vertebral em escolares de 10 a 16 anos de idade do município de Tangará, SC, 2004 ( $n=344)$.

Table 1 - Prevalence of backbone postural changes in 10 to 16 year-old schoolchildren from the city of Tangará, SC, 2004 ( $n=344)$.

\begin{tabular}{lcc}
\hline Alterações Posturais de Coluna Vertebral & $\begin{array}{c}\text { Prevalência } \\
\mathrm{n}(\%)\end{array}$ & IC 95\% \\
\hline Hiperlordose & $70(20,3)$ & $16,1-24,5$ \\
Hipercifose & $38(11,0)$ & $7,7-14,3$ \\
Escoliose funcional & $11(3,2)$ & $1,4-5,0$ \\
Cifoescoliose funcional & $06(1,7)$ & $0,4-3,0$ \\
Escoliose estrutural & $05(1,5)$ & $0,3-2,7$ \\
Cifoescoliose estrutural & $04(1,2)$ & $0,06-2,34$ \\
Lordoescoliose funcional & $02(0,6)$ & $0,06-2,34$ \\
Lordoescoliose estrutural & $02(0,6)$ & $0,9-2,1$ \\
Qualquer alteração & $97(28,2)$ & $12,4-32,9$ \\
\hline
\end{tabular}


Tabela 2 - Presença de qualquer alteração postural de coluna vertebral, hiperlordose e hipercifose e fatores associados em escolares de 10 a 16 anos de idade do município de Tangará, SC, 2004.

Table 2 - Presence of any backbone postural changes, high concavity and high kyphosis and related factors in 10 to 16 yearold schoolchildren of the city of Tangará, SC, 2004.

\begin{tabular}{|c|c|c|c|c|c|c|c|c|c|}
\hline \multirow[t]{2}{*}{$\begin{array}{l}\text { Variável } \\
\text { (Categorias) }\end{array}$} & \multicolumn{2}{|c|}{$\begin{array}{c}\text { Presença de Alteração } \\
\text { Postural de } \\
\text { Coluna Vertebral }\end{array}$} & \multirow[b]{2}{*}{$\mathrm{p}^{*}$} & \multicolumn{2}{|c|}{$\begin{array}{l}\text { Presença de } \\
\text { Hiperlordose }\end{array}$} & \multirow[b]{2}{*}{$\mathrm{p}^{*}$} & \multicolumn{2}{|c|}{$\begin{array}{l}\text { Presença de } \\
\text { Hipercifose }\end{array}$} & \multirow[b]{2}{*}{$\mathrm{p}^{*}$} \\
\hline & $\operatorname{sim}(\%)$ & Não (\%) & & $\operatorname{sim}(\%)$ & Não (\%) & & $\operatorname{sim}(\%)$ & Não (\%) & \\
\hline Idade (anos) & & & 0,520 & & & 0,110 & & & 0,468 \\
\hline$<\mathrm{ou}=12$ & $54(29,7)$ & $128(70,3)$ & & $43(23,6)$ & $139(76,4)$ & & $18(9,9)$ & $164(90,1)$ & \\
\hline 13 a 16 & $43(26,5)$ & $119(73,5)$ & & $27(16,7)$ & $135(83,3)$ & & $20(12,3)$ & $142(87,7)$ & \\
\hline Sexo & & & 0,425 & & & 0,993 & & & 0,971 \\
\hline Masculino & $49(30,2)$ & $113(79,8)$ & & $33(20,4)$ & $129(79,6)$ & & $18(11,1)$ & $144(88,9)$ & \\
\hline Feminino & $48(26,4)$ & $134(73,6)$ & & $37(20,3)$ & $145(79,7)$ & & $20(11,0)$ & $162(89,0)$ & \\
\hline Renda familiar (reais) & & & 0,206 & & & 0,440 & & & 0,100 \\
\hline$<\mathrm{ou}=700,00$ & $45(33,8)$ & $88(66,2)$ & & $33(19,6)$ & $135(80,4)$ & & $16(9,5)$ & $152(90,5)$ & \\
\hline 701,00 a 2500,00 & $45(26,8)$ & $123(73,2)$ & & $31(23,3)$ & $102(76,7)$ & & $21(15,8)$ & $112(84,2)$ & \\
\hline Escolaridade pai & & & 0,380 & & & 0,400 & & & 0,236 \\
\hline$<\mathrm{ou}=4$ anos & $55(29,7)$ & $130(70,3)$ & & $39(21,1)$ & $146(78,9)$ & & $23(12,4)$ & $162(87,6)$ & \\
\hline$>4$ anos & $32(24,1)$ & $101(75,9)$ & & $23(17,3)$ & $110(82,7)$ & & $11(8,3)$ & $122(91,7)$ & \\
\hline Escolaridade mãe & & & 1,000 & & & 0,606 & & & 0,217 \\
\hline$<\mathrm{ou}=4 \mathrm{anos}$ & $60(27,9)$ & $155(72,1)$ & & $44(20,5)$ & $171(79,5)$ & & $21(9,8)$ & $194(90,2)$ & \\
\hline$>4$ anos & $32(27,6)$ & $84(72,4)$ & & $20(18,1)$ & $95(81,9)$ & & $16(13,8)$ & $100(86,2)$ & \\
\hline Altura média (m) & & & 0,008 & & & 0,003 & & & 0,731 \\
\hline$<\mathrm{ou}=1,54$ & $60(34,9)$ & $112(65,1)$ & & $46(26,7)$ & $126(73,3)$ & & $20(11,6)$ & $152(88,4)$ & \\
\hline$>1,54$ & $37(21,5)$ & $135(78,5)$ & & $24(14,0)$ & $148(86,0)$ & & $18(10,5)$ & $154(89,5)$ & \\
\hline Peso/mediana (Kg) & & & 0,055 & & & 0,028 & & & 0,620 \\
\hline$<\mathrm{ou}=45$ & $58(33,0)$ & $118(67,0)$ & & $44(25,0)$ & $132(75,0)$ & & $18(10,2)$ & $158(89,8)$ & \\
\hline$>45$ & $39(23,2)$ & $129(76,8)$ & & $26(15,5)$ & $142(84,5)$ & & $20(11,9)$ & $148(88,1)$ & \\
\hline IMC & & & 0,143 & & & 0,220 & & & 0,748 \\
\hline$<\mathrm{ou}=19$ & $65(31,1)$ & $144(68,9)$ & & $47(22,5)$ & $162(77,5)$ & & $24(11,5)$ & $185(88,5)$ & \\
\hline$>19$ & $32(23,7)$ & $103(76,3)$ & & $23(17,0)$ & $112(83,0)$ & & $14(10,4$ & $121(89,6)$ & \\
\hline Dominância & & & 0,804 & & & 0,587 & & & 1,000 \\
\hline Destro & $92(28,5)$ & $231(71,5)$ & & $67(20,7)$ & $256(79,3)$ & & $36(11,1)$ & $287(88,9)$ & \\
\hline Sinistro & $5(23,8)$ & $16(76,2)$ & & $3(14,3)$ & $18(85,7)$ & & $2(9,5)$ & $19(90,5)$ & \\
\hline
\end{tabular}

com até $1,54 \mathrm{~m}$ apresentaram estatisticametne mais alterações posturais se comparados com seus colegas mais altos $(\mathrm{p}=0,008)$. A variável peso corporal mostrou-se no limite da associação, direcionada para aqueles com peso inferior a 45 $\mathrm{kg}(\mathrm{p}=0,055)$. Na mesma tabela, pode-se observar ainda que a prevalência de hiperlordose mostrou-se estatisticamente associada ao peso e a altura dos escolares. Escolares de mais baixa estatura (menores que $1,54 \mathrm{~m})(\mathrm{p}=0,003)$ e menos pesados (menos de $45 \mathrm{Kg})(\mathrm{p}=0,028)$ apresentaram mais alterações tipo hiperlordose que seus colegas. Já a prevalência de hipercifose não se mostrou estatisticamente associada às variáveis estudadas.

\section{Discussão}

A prevalência de alterações posturais de coluna vertebral encontrada entre es- 
colares de Tangará, SC $(28,2 \%)$ foi relativamente baixa, se comparada à prevalência de 90,0\% observada em escolas do Rio Grande do Sul ${ }^{9}$. A hiperlordose foi a alteração mais prevalente (20,3\%) corroborando também o estudo anteriormente citado. Já na cidade de Marília, SP, a prevalência de alteração do tipo hiperlordose foi de $35,1 \%$ também entre escolares ${ }^{10}$. Em um outro estudo realizado com escolares de 7 a 12 anos em uma escola pública de São Paulo, constatou-se a prevalência de hiperlordose lombar de 73,8\%, escoliose de $23,8 \%$ e hipercifose torácica de $9,5 \%{ }^{11}$.

Outro achado desse estudo foi a proporção significativa de hipercifose $(11,0 \%)$ entre os indivíduos, embora inferior aos valores encontrados em Catanduva, SP. Verificou-se que entre escolares de 6 a 17 anos dessa cidade, $20,9 \%$ apresentavam hipercifose, sendo que $14,9 \%$ apresentavam hipercifose associada a uma hiperlordose e $6,9 \%$ apresentavam hipercifose associada à protrusão de cabeça ${ }^{4}$.

As alterações do tipo escoliose funcional $(3,2 \%)$ estiveram mais presentes do que as escolioses com evidências estruturais $(1,5 \%)$. As prevalências de escolioses encontradas em Tangará, SC, se enquadram nas prevalências esperadas de 1 a $13 \%$ em todo o mundo ${ }^{12}$. Em contrapartida, outros autores descrevem que a prevalência das escolioses varia de $1 \%$ a $21 \%$ e atribuem essa grande diferença ao fato de serem usados diferentes critérios de detecção e diagnóstico ${ }^{13}$. Embora com baixas prevalências, se comparadas à hipercifose e hiperlordose, estas alterações devem ser tratadas com cuidado para melhorar a qualidade de vida do escolar, prevenindo sua progressão, pois podem se tornar incapacitantes na fase adulta.
Em relação aos fatores associados, este estudo mostrou associações das alterações posturais com menor estatura e menor peso corporal. Robergs, Roberts ${ }^{14}$ salientam que pode haver diferenças de prevalência de alterações posturais entre escolares de mesma idade, sexo, peso e altura em decorrência do nível de maturação dos padrões de crescimento e desenvolvimento. Em decorrência da inadequada ergonomia escolar, os escolares em fase de crescimento e desenvolvimento, a despeito do peso e altura, tendem a apresentar alterações posturais após alguns anos de freqüência à escola ${ }^{15}$.

Como se observou nesse tipo de estudo, a maioria das alterações posturais pode ser considerada reversível. Nesse contexto, ganham importância campanhas de promoção de saúde que objetivem a adoção de estilos de vida e posturas mais saudáveis, incluindo a prevenção e o tratamento das alterações posturais. Os escolares com alterações posturais consideradas potencialmente mais incapacitantes, como as escolioses com evidências estruturais, necessitam de encaminhamento ao sistema de saúde do município. Através de exames complementares, a equipe de saúde poderá obter dados mais precisos e detalhados em relação às alterações internas da coluna vertebral do indivíduo, possibilitando traçar um diagnóstico clínico acurado. Esse diagnóstico poderá facilitar o tratamento fisioterapêutico, fundamental para o tratamento da progressão das alterações.

Pode-se concluir que as alterações posturais de coluna mais prevalentes foram a hiperlordose e a hipercifose. A estatura e o peso corporal foram as variáveis que se mostraram estatisticamente associadas à prevalência.

\section{Referências}

1. Adler NS, Csongradi J, Bleck EE. School Screening for scoliosis. WJ Med 1984; 141: 631-3.

2. Kavalco TF. A manifestação de alterações posturais em crianças de primeira a quarta séries do ensino fundamental e sua relação coma ergonomia escolar. Rev Bras Fisioterapia 2000; 2(4).

3. Bruschini S. Ortopedia Pediátrica. 2a ed., São Paulo: Atheneu; 1998. 
4. Vilarinho RMA. Incidência de hipercifose como alteração postural em escolares de 6 a 17 anos em uma escola pública municipal da cidade Catanduva. Rev Acta Fisiátrica 2002; 1(9).

5. Ferronatto A, Landotti CT, Silveira RP. A incidência de alterações do equilíbrio estático da cintura escapular em crianças entre 7 a 14 anos. Rev Movimento1998; 9: 25-30.

6. Dickson RA. Scoliosis in the community. Br Med J 1983; 286: 615-8.

7. Kendall FP, McCreary EK. Músculos: Provas e Funções. $4^{\mathrm{a}}$ ed, São Paulo: Manole; 1995.

8. Peres MA, Traebert J, Marcenes. Calibração de examinadores para estudos epidemiológicos da cárie dentária. Cad Saúde Pública 2001; 17:153-9.

9. Chaffin D, Andersson GBJ, Martin BJ. Biomecânica Ocupacional. Belo Horizonte: Ergo; 2001.

10. Marrele EP. Avaliação postural em crianças de primeira a quarta série de Marília-SP. VI Simpósio de Iniciação Científica-UNIMAR; 2004.
11. Bertoldi LF, Bianchi PC, Borges NP, Carrara E, Carvalho SM, Castellani TM. Avaliação e orientação postural em escolares de 7 a 12 anos do Colégio Estadual Jardim Piza Roseira [online]. Disponível em:<http://www.ccs.br/ olhomagico/peepin98/Gim16.html [março 2005].

12. Brooks HL. Scoliosis: a prospective epidemiological study. J Bone Joint Surg 1975; 57(7): 968-72.

13. Karachalios T, Sofianos J, Roidis N, Sapkas G, Korres D, Nikolopoulos K. Ten year follow-up evaluation of a school screening program for scoliosis: is the forward-bending test an accurate diagnostic criterion for the screening of scoliosis? Spine 1999; 24 (12): 2318-24.

14. Robergs R, Roberts SO. Princípios fundamentais de fisiologia do exercício para aptidão, desempenho e saúde. São Paulo: Phorte; 2002.

15. Mandal AC. Investigation of the lumbar flexion of office workers. The ergonomics of working postures. London and Philadelphia : Taylor \& Francis. 1986. p. 345-354.

Recebido em: 23/09/05

Versão reformulada reapresentada em: 06/12/05

Aprovado em: 07/12/05 